\title{
Tiefe Hirnstimulation bei Morbus Parkinson
}

Petyo Nikolov, Stefan Jun Groiss, Alfons Schnitzler

\begin{abstract}
Die tiefe Hirnstimulation ist eine etablierte und evidenzbasierte Therapieoption für die Behandlung des fortgeschrittenen Morbus Parkinson. Es handelt sich um eine symptomatische Behandlung mit dem primären Ziel, die Lebensqualität des Patienten zu verbessern. Der operative Eingriff gilt als relativ komplikationsarm.
\end{abstract}
ABKÜRZUNGEN
GPi Globus pallidus internus
IPG Implantierbarer Generator
STN Nucleus subthalamicus
THS Tiefe Hirnstimulation

\section{Einleitung}

Der Morbus Parkinson ist mit einer Inzidenz von 100-200 Neuerkrankungen pro 100000 Einwohnern pro Jahr in Deutschland die zweithäufigste neurodegenerative Erkrankung. Die Prävalenz von 0,3\% in der Gesamtbevölkerung steigt mit zunehmendem Alter an und beträgt $1 \%$ in der Altersgruppe über 65 Jahre [1]. Aufgrund des demografischen Wandels mit steigender Lebenserwartung wird die Häufigkeit der Parkinson-Krankheit künftig weiter zunehmen.

Klinisch ist der Morbus Parkinson charakterisiert durch die motorischen Kardinalsymptome Bradykinese, Rigor und/ oder Tremor sowie durch eine Vielzahl nichtmotorischer Symptome.

Neuropathologisch ist der Untergang dopaminerger Neurone in der Substantia nigra pars compacta für die Ausbildung der motorischen Symptomatik verantwortlich. Die medikamentöse Behandlung der motorischen Symptome zielt daher im Wesentlichen auf die Verringerung des nigrostriatalen dopaminergen Defizits ab.

\section{FALLBEISPIEL}

\section{Patientenvorstellung}

Herr M., 41 Jahre alt, stellt sich mit einem seit 9 Jahren bestehenden idiopathischen Parkinson Syndrom (Hoehn und Yahr-Stadium III) vor. Parkinson-Medikation: Insgesamt 1000/250 mg L-Dopa/Benserazid, aufgeteilt auf 6 Einzeldosen. Dazu 50 mg Opicapon und 100 mg Amantadin täglich. Pramipexol retard $(2,1 \mathrm{mg} / \mathrm{d})$ wurde aufgrund von Impulskontrollstörungen kürzlich ausschleichend abgesetzt.

Der Patient klagt unter der aktuellen Medikation über hypokinetische Phasen, die 50\% der Tageszeit bestehen und z. T. abrupt im Sinne von Sudden-Off Zuständen einsetzen. Dazu kommt es zu regelhaften peak-of-dose Hyperkinesen. An vegetativer Symptomatik bestehen ein leichter orthostatischer Schwindel und eine Obstipation. Die Stimmung schwankt im Sinne von non-motor fluctuations. OFF-assoziiert kommt es regelmäßig zu Panikattacken mit vegetativer Begleitsymptomatik. Konzentration und Gedächtnis sind weitestgehend stabil. Impulskontrollstörungen in Form von Spielsucht und Hypersexualität sind seit Absetzen von Pramipexol rückläufig aber noch nicht vollständig abgeklungen. Es stellt sich die Frage, ob eine tiefe Hirnstimulation in diesem Fall indiziert ist.

\section{Grenzen der medikamentösen dopaminergen Therapie}

Seit der Einführung vor 50 Jahren gilt L-Dopa als wirksamste und gleichzeitig sichere Substanz in der symptomatischen Behandlung des M. Parkinson, sodass fast jeder Patient im Laufe seiner Erkrankung auf eine regelmäßige Einnahme von L-Dopa angewiesen ist. 
Merke

Unter der dopaminergen Behandlung und mit

Fortschreiten der Erkrankung treten hypokinetische

Wirkfluktuationen und Dyskinesen auf, die die

Lebensqualität zunehmend beeinträchtigen.

Darüber hinaus kann es zu neuropsychiatrischen Nebenwirkungen wie Impulskontrollstörungen, Schlafstörungen, dem L-Dopa-Dysregulations-Syndrom und Halluzinationen kommen. Motorische Spätkomplikationen und Nebenwirkungen können die Effektivität der medikamentösen Therapie deutlich einschränken und sich enorm auf die Lebensqualität der Betroffenen auswirken. In der Tat entwickeln $50 \%$ aller Parkinson-Patienten bereits nach den ersten drei Jahren der Behandlung („,honeymoon phase“) erste motorische Fluktuationen [2]. Als Risikofaktoren gelten $[3,4]$ :

- hohe initiale L-Dopa Dosis

- längere Therapiedauer

- männliches Geschlecht

- niedriger BMI

- Auftreten der Erkrankung im jüngeren Alter

Motorische Fluktuationen und Dyskinesien stellen in fortgeschrittenen Stadien der Parkinson-Erkrankung eine der wesentlichen therapeutischen Herausforderungen dar. Ist eine zufriedenstellende Pharmakotherapie nicht mehr möglich, sollte die Indikation zur Hirnstimulationsbehandlung geprüft werden.

\section{Tiefe Hirnstimulation (THS) - Einleitung}

Bei der THS erfolgt eine Implantation von Elektroden im Rahmen eines funktionellen stereotaktischen Eingriffs in der Regel bilateral in eine subkortikale Zielstruktur, die abhängig von der zu behandelnden Erkrankung gewählt wird. Die Stimulationselektroden werden durch ein subkutan verlaufendes Extensionskabel mit einem infraklavikulär oder abdominal implantierten Impulsgenerator (IPG) verbunden. Die Behandlung des fortgeschrittenen M. Parkinson wurde mit dem bis heute verwendeten Zielpunkt Nucleus subthalamicus (STN) erstmals 1994 von Benabid beschrieben [5], nachdem im Tierexperiment nachgewiesen worden war, dass dieser Basalganglien-Kern bei der Parkinson-Erkrankung eine pathologische Überaktivität zeigt. Die STN-THS hat sich seither zu einer sehr wirksamen, sicheren und evidenzbasierten Therapie des fortgeschrittenen M. Parkinson etabliert. Neben dem STN wird insbesondere in den USA auch der Globus pallidus internus (GPi) als Zielgebiet für die Stimulation gewählt. Zurzeit sind in Deutschland THS-Systeme von 3 Herstellern (Abbott, Boston Scientific, Medtronic) zur Therapie zugelassen. Technische Fortschritte haben in den vergangenen Jahren zu Verbesserungen der Systeme geführt, die höhere Freiheitsgrade in der therapeutischen Einstellung der Schrittmacher erlauben, gleichzeitig aber auch eine größere Expertise der behandelnden Neurologen verlangen.

\section{Wirkmechanismen der THS des Nucleus subthalamicus (STN-THS)}

Ursprünglich wurde als wesentlicher Wirkmechanismus der THS eine neuronale Inhibition des Zielgebietes abgenommen, da mit einer Läsion ein vergleichbarer Effekt auf Symptomebene erzielt werden kann wie mit der Stimulation. Neuere Untersuchungen haben jedoch gezeigt, dass es durch die Stimulation zu einer direkten Erregung von Axonen kommt [6]. Tierexperimentelle Untersuchungen legen nahe: als Mechanismus der STN-THS spielt die antidrome Aktivierung des hyperdirekten Fasertrakts zwischen STN und Motorcortex mit resultierender Hemmung pathologisch gesteigerter $\beta$-Oszillationen vermutlich eine entscheidende Rolle [7]. Auch bei Parkinson Patienten konnte gezeigt werden, dass die STN-THS mit einer Suppression verstärkter $\beta$-Oszillationen im STN einhergeht und diese mit der Besserung von Akinesie und Rigor korreliert [1, 8]. Die genauen Mechanismen der STN-THS auf molekularer, zellulärer, lokaler und Netzwerkebene sind jedoch noch weitgehend ungeklärt und aktueller Gegenstand wissenschaftlicher Untersuchungen.

Merke

STN und Globus pallidus internus (GPi) sind Zielgebiete für die THS bei Parkinson, wobei in Europa der STN der bevorzugte Zielpunkt ist. Die STN-THS führt zur Suppression gesteigerter $\beta$-Oszillationen.

\section{Praktisches Vorgehen}

Die Durchführung einer erfolgreichen STN-THS beruht auf mehreren Säulen und erfordert die interdisziplinäre Kooperation in einem spezialisierten Team aus Neurologen, funktionellen Neurochirurgen, Neuropsychologen, Psychiatern und spezialisierten Pflegekräften. Sie fängt mit der korrekten Indikationsstellung und Auswahl der richtigen Patienten an und beinhaltet das perioperative Management sowie die postoperative Langzeitbetreuung.

\section{FALLBEISPIEL}

\section{Befunde}

Der Patient wurde stationär zur Abklärung einer THS Indikation aufgenommen. Folgende Befunde liegen vor:

- MRT: altersentsprechender unauffälliger Befund, insbesondere keine relevante Atrophie.

- L-Dopa-Test: gutes Ansprechen auf L-Dopa. UPDRS III im Med-OFF: 34 Punkte, Med-ON: 14 Punkte.

- Neuropsychologie: kein Hinweis auf eine klinisch relevante kognitive Beeinträchtigung. MDRS: 135/144 Punkte; MoCA: 27/30 Punkte. 


\section{Patientenselektion}

Für die Indikation zur STN-THS muss das Vorliegen eines idiopathischen Parkinson-Syndroms mit medikamentös nicht mehr befriedigend behandelbaren motorischen Fluktuationen, Dyskinesien und/oder Tremor gesichert sein. Atypische oder sekundäre Formen müssen ausgeschlossen werden, da sie von einer THS nicht profitieren.

Entsprechend gilt das prinzipielle medikamentöse Ansprechen von Akinese und Rigor auf L-Dopa, definiert als eine Verbesserung im UPDRS III um mindestens $30 \%$ im einzeitigen L-Dopa-Test, als wichtiges Einschlusskriterium und gleichzeitig als Prädiktor für das therapeutische Ansprechen auf die STN-THS. Ausgenommen von dieser Bedingung ist das Zielsymptom Tremor, da die STN-THS auch bei fehlender Wirkung der dopaminergen Medikation eine gute Tremorsuppression zeigt.

Aufgrund neuerer Daten können das Vorliegen nichtmotorischer neuropsychiatrischer Fluktuationen wie OFF-assoziierte Depressions- und Angstzustände sowie nebenwirkungsbedingte Impulskontrollstörungen im Rahmen der dopaminergen Therapie als unterstützende Kriterien für die Indikationsstellung betrachtet werden [9].

\section{Einfluss des Patientenalters}

Weitere Kriterien zur THS-Indikation sind das Patientenalter und die Erkrankungsdauer. Hinsichtlich des Alters des Patienten ist das individuelle biologische Alter entscheidend. Jüngere Patienten scheinen im Vergleich zu älteren Patienten von einer signifikant höheren Lebensqualität nach THS zu profitieren [10]. Es gibt allerdings keine festen Empfehlungen in Bezug auf eine starre Altersgrenze. Entscheidungen sind individuell zu treffen. Die Indikation einer STN-THS jenseits des 75. Lebensjahrs sollte jedoch sehr kritisch überprüft werden, da sich ein altersentsprechend reduzierter Allgemeinzustand durch die Operation dauerhaft verschlechtern kann und sich z. B. eine beginnende aber bislang klinisch noch kompensierte kognitive Beeinträchtigung durch den Eingriff akzentuieren kann.

\section{Weitere Selektionskriterien}

Hinsichtlich der Erkrankungsdauer wurden in den ersten 2 Dekaden der THS Anwendung typischerweise Patienten behandelt, die im Mittel 10-14 Jahre erkrankt waren, tägliche OFF-Zeiten zwischen 4 und 8 Stunden hatten und sich somit in einem fortgeschrittenen Stadium befanden. Eine EARLYSTIM-Studie [11], hat jedoch gezeigt, dass die STN-THS auch bei Patienten mit einer mittleren Krankheitsdauer von im Schnitt 7 Jahren einer bestmöglichen medikamentösen Therapie bezüglich des primären Outcome Parameters Lebensqualität und weiterer Parameter signifikant überlegen ist. Demzufolge hat sich das Zeitfenster für STN-THS zum mittleren Krankheitsstadium hin erweitert. Zu berücksich- tigen sind dabei jedoch die spezifischen Einschlusskriterien der EARLY-STIM-Studie.

EINSCHLUSSKRITERIEN DER EARLY-STIMSTUDIE

- Erkrankungsdauer >4 Jahre

- keine Hinweise auf ein sekundäres oder atypisches Parkinsonsyndrom

- exzellentes Ansprechen auf Levodopa (> 50 \% im standardisierten L-Dopa-Test)

- mindestens leichte Wirkfluktuationen

- keine relevante Komorbidität

- Zugang zu einem Zentrum mit einem erfahrenen multidisziplinären Team hinsichtlich Patientenselektion, Operation, Programmierung und Nachsorge

Als Kontraindikationen für eine STN-THS gelten generell schwere Allgemeinerkrankungen, erhöhte Blutungsneigung, manifeste psychiatrische Erkrankungen wie z. B. eine medikamentös schwer behandelbare Depression, Demenz, und neurochirurgische Kontraindikationen.

Merke

Zum Ausschluss einer Demenz bzw. einer relevanten kognitiven Störung werden die Mattis Demenz Rating Scale (MDRS, Cut-Off-Score >130) sowie das Montreal Cognitive Assessement (MoCA, Cut-Off-Score > 26) angewandt.

Neben diesen Faktoren sollte unbedingt auch die Zielsetzung der THS klar kommuniziert werden. Eine realistische Erwartungshaltung des Patienten und seines sozialen Umfelds ist von großer Bedeutung für ein befriedigendes Gesamtergebnis. Die THS ist eine symptomatische Behandlung und das übergeordnete Ziel die Verbesserung der Lebensqualität. Daher sollten die für den Patienten entscheidenden Faktoren der Lebensqualitätsminderung identifiziert und deren Ansprechen auf die THS bewertet werden. Bei korrekter Indikationsstellung ist die Lebensqualität nicht nur ein wichtiger Outcome-Parameter, sondern auch ein Outcome-Prädiktor der STN-THS. Je geringer die Lebensqualität - gemessen mit dem PDQ39 - vor der Operation ist, desto größer ist die Verbesserung der Lebensqualität nach 2 Jahren STN-THS [11]. 


\section{INFOBOX}

\section{Indikationskriterien}

- Vorliegen eines idiopathischen Parkinson Syndroms mit einem der folgenden medikamentös nicht befriedigend behandelbaren Symptome:

- Behindernde Wirkungsfluktuationen/ Dyskinesien

- Therapierefraktärer Tremor

- Dopamin-assoziierte, therapielimitierende Nebenwirkungen

- Gutes Ansprechen der Symptome auf L-Dopa; Tremor zählt nicht dazu (UPDRS III Besserung $>33 \%$ bei L-Dopa-Test).

- Realistische Erwartungshaltung und stabile soziale Situation

- Unauffälliger MRT Befund

- Zugang zu einem spezialisierten Zentrum mit erfahrenem interdisziplinärenTeam

\section{Ausschlusskriterien}

- Atypische Parkinsonsyndrome

- Schwere Allgemeinerkrankungen

- Relevante kognitive Defizite (MDRS < 130 Punkte)

- Relevante chirurgische Risikofaktoren (z. B. Blutungsneigung)

- Relevante pathologische Veränderungen in MRT

- Schwere Depression

Generell sprechen alle L-Dopa-responsiven Symptome auf die STN-THS an. Hypodopaminerge motorische und nichtmotorische Wirkfluktuationen können deutlich reduziert werden. Ferner werden hyperdopaminerge motorische Symptome wie Dyskinesien und nichtmotorische Symptome wie Impulskontrollstörungen aufgrund der durch die STN-THS mögliche Reduktion der dopaminergen Medikation gebessert. Insgesamt resultiert daraus eine Verlängerung der Zeiten in einem guten motorischen und psychischen ON-Zustand. Nicht L-Dopa-responsive Symptome wie posturale Instabilität im medikamentösen ON sprechen dagegen nicht auf die STN-THS an.

\section{Perioperatives Management}

Voraussetzung für eine erfolgreiche Elektrodenimplantation sind die präzise Zielpunktlokalisation und Trajektplanung basierend auf der individuellen Patientenanatomie. Dazu werden heute in der Regel MRT und stereotaktische CT-Bilder fusioniert ( $>$ Abb. 1), um die im MRT identifizierten Zielpunkte und die Elektrodentrajekte in das stereotaktische Koordinatensystem überführen zu können. Intra-

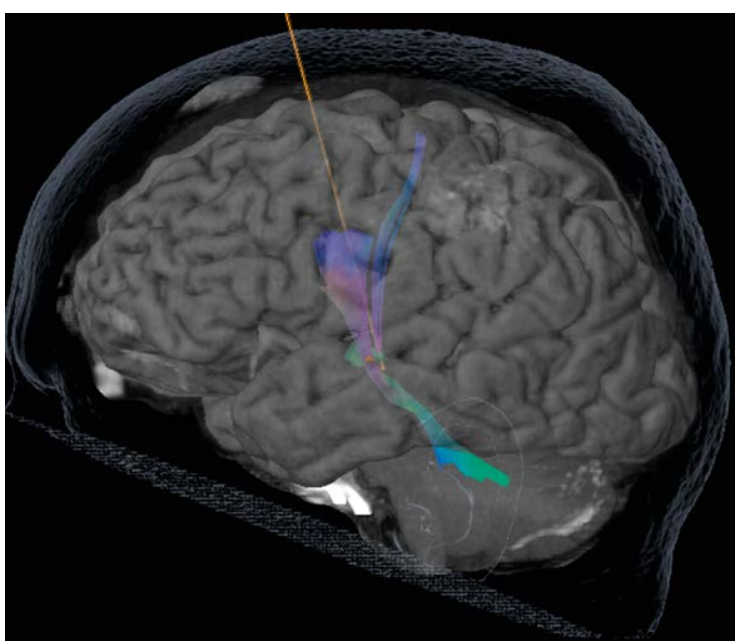

- Abb. 1 Darstellung einer THS-Elektrode im STN anhand fusionierter DTI-MRT-/CT-Aufnahmen.

operativ kann die anatomische Zielpunktlokalisation elektrophysiologisch und klinisch durch Mikroelektrodenableitungen (MER) und intraoperative Teststimulation unterstützt werden. Mittels MER werden die Grenzen des STN ermittelt, indem auf verschiedenen Höhen entlang des Trajekts neuronale Aktivität abgleitet wird. Mit der intraoperativen Teststimulation, die im Wachzustand des Patienten erfolgen muss, werden therapeutische Effekte und mögliche Nebenwirkungen im Bereich des angesteuerten Zielpunktes überprüft [1]. Die Ergebnisse von MER und Teststimulation können in manchen Fällen zu einer Anpassung des gewählten anatomischen Zielpunktes führen. Die finale Platzierung der THS-Elektrode wird also auf der Basis der anatomischen Zielpunktlokalisation unter Berücksichtigung der Ergebnisse der intraoperativen Mikroableitungen und Teststimulation gewählt.

\section{PERIOPERATIVES RISIKO}

Die THS-Operation gilt generell als relativ komplikationsarm. Die Häufigkeit perioperativer Komplikationen wurde in einer Metaanalyse mit 728 Patienten wie folgt beschrieben [12]: Intraoperativ: symptomatische intrazerebrale Blutung ( $1,1 \%)$; ischämischer Schlaganfall $(0,4 \%)$; Hypotension (0,3\%); epileptischer Anfall (0,3\%). Postoperativ: Infektion (1,7\%); Elektroden Dislokation (1,7\%); Hardware-assoziierter Dyskomfort $(1,1 \%)$. 


\section{FALLBEISPIEL}

\section{Behandlung}

Es erfolgte eine komplikationslose Implantation der Elektroden in den STN und des IPG.

Im monopolaren Review wurden alle Elektrodenkontakte direktional sowie omnidirektional ausgetestet, um die besten Kontakte und die initialen Parameter für die chronische therapeutische Stimulation des Patienten zu ermittelt. Während des monopolaren Reviews wurden folgende Stimulationsparameter konstant gehalten: monopolar; IPG (+); Pulsbreite $60 \mu$ s und Frequenz $130 \mathrm{~Hz}$. Die Intensität wurde dabei variiert und die therapeutischen, sowie die Nebenwirkungsschwellen dokumentiert. Als beste Kontakte wurden diejenigen mit dem größten therapeutischen Fenster (Differenz zwischen Nebenwirkungsschwelle und therapeutischer Schwelle) definiert. Im Verlauf zeigte sich unter der chronischen Stimulation eine gute Symptomkontrolle mit Besserung des Gangbildes sowie Reduktion der Fluktuationen und Dyskinesien mit entsprechender Zunahme der ON-Zeiten ohne Dyskinesien. Die dopaminerge Medikation konnte von $1000 \mathrm{mg}$ auf $600 \mathrm{mg}$ L-Dopa reduziert werden.

\section{Stimulationsparameter bei Entlassung}

STN links: Kontakt 2; Segment B; Intensität 1,4 mA; Pulsbreite $60 \mu \mathrm{s}$, Frequenz $130 \mathrm{~Hz}$

STN rechts: Kontakt 10; Segment A; Intensität

1,5 mA; Pulsbreite $60 \mu \mathrm{s}$, Frequenz $130 \mathrm{~Hz}$

Medikation: 600/150mg L-Dopa/Benserazid täglich, verteilt auf vier Tagesdosen. Dazu 50 mg Opicapon. Anschließend wurde eine erneute stationäre Aufnahme zur Kontrolle der Stimulationsparameter nach 3 Monaten empfohlen.

\section{Programmierung}

Nach der erfolgreichen Elektroden- und IPG-Implantation erfolgt die Programmierung der Parameter für die chronische therapeutische Stimulation. Nach der Operation tritt häufig ein Setz- bzw. Mikroläsionseffekt mit vorübergehender Symptomlinderung ein. Daher empfiehlt es sich, unmittelbar postoperativ zunächst eine vorläufige Auswahl der Stimulationskontakte für die therapeutische Stimulation auf der Basis der anatomischen Zielpunktlokalisation vorzunehmen.

\section{Test der Stimulationskontakte}

Nach Abklingen des Setzeffektes können die Stimulationskontakte dann detailliert in sogenannten monopolaren Reviews ausgetestet werden. Nach Absetzen der dopa- minergen Medikamente für mindestens 12 Stunden wird dabei der klinische Effekt der Stimulation jedes einzelnen Elektrodenkontakts separat ausgetestet. Dabei wird die Wirkung auf motorische Symptome auf der Basis standardisierter Scores wie dem UPDRS-III bewertet. Nebenwirkungen werden individuell dokumentiert. Der Effekt der Stimulation hängt primär von der Größe und Form des erzeugten elektrischen Feldes ab. Diese können über die Parameter Intensität, Frequenz, Pulsbreite, Direktionalität und Polarität gesteuert werden.

Merke

Die Zahl theoretisch möglicher Kombinationen von Stimulationsparametern ist viel zu hoch, um sie in der Praxis austesten zu können. Daher geht man beim monopolaren Review nach einem etablierten Algorithmus vor [13], bei dem die Polarität (monopolar; IPG (+)), die Pulsbreite $(60 \mu \mathrm{s})$ und Frequenz $(130 \mathrm{~Hz})$ konstant gehalten werden und lediglich die Intensität variiert wird.

Bei herkömmlichen nicht-segmentierten Elektroden und omnidirektionaler Stimulation wird zur Austestung initial eine Schrittgröße von 0,5 mA gewählt. Bei direktionaler Stimulation mit segmentierten Elektroden führt eine gegebene Stromamplitude im Vergleich zur omnidirektionalen Stimulation zu einer höheren Ladungsdichte am Kontakt. Daher sind niedrigere Schwellenwerte für eine klinische Verbesserung einerseits und Nebenwirkungen andererseits zu erwarten. Aus diesem Grund sollte primär eine kleinere Schrittgröße von 0,2 mA bei der Erhöhung der Stimulationsamplitude gewählt werden. Folgend kann eine feinere Adjustierung mit Schritten von $0,1 \mathrm{~mA}$ vorgenommen werden, um die therapeutische Schwelle genauer zu definieren.

\section{Merke \\ Als therapeutische Schwelle wird die Stimulationsintensität verstanden, die eine klinisch relevante Symptombesserung hervorruft. Die Nebenwirkungsschwelle liegt bei der Intensität vor, die zu persistierenden Nebenwirkungen führt.}

Danach wird die Intensität über die therapeutische Schwelle hinaus weiter erhöht, um die Nebenwirkungsschwelle zu bestimmen. Die Differenz zwischen therapeutischer und Nebenwirkungsschwelle wird als therapeutisches Fenster bezeichnet. Der Kontakt mit dem größten therapeutischen Fenster sollte für die chronische THS gewählt werden.

\section{Chronische Stimulation}

Bei der Anwendung der chronischen Stimulation sollte die Stimulationsamplitude langsam erhöht und gleichzeitig eine schrittweise Reduktion der dopaminergen Medikation vorgenommen werden. Eine zu schnelle Erhöhung der 
Stimulationsintensität kann bei der STN-THS Dyskinesien auslösen, die manchmal erst mit einer Verzögerung von Stunden auftreten. Generell ist es wichtig zu beachten, dass im monopolaren Review nur die sofortigen Nebenwirkungen erfasst werden. Um verzögert auftretende Nebenwirkungen wie Dysarthrie oder Gangataxie frühzeitig zu erkennen, ist eine entsprechende ambulante Anbindung des Patienten erforderlich.

Merke

Die Rheobase ist die kleinste Reizstärke, die beim kontinuierlichen Reiz notwendig ist, um eine vordefinierte Faser zu aktivieren. Die Chronaxie ist die kürzeste Zeit, über die ein Reiz doppelter Rheobase einwirken muss, um dieselbe Faser zu aktivieren.

Die gängige Pulsbreite für STN-THS beträgt $60 \mu \mathrm{s}$. Durch Veränderung der Pulsbreite lassen sich Fasern, die unterschiedliche Chronaxien aufweisen, selektiver stimulieren. Daher kann bei niedrigen Nebenwirkungsschwellen die Anwendung von Impulsen kurzer Dauer ( $<60 \mu \mathrm{s}$ ) das therapeutische Fenster erweitern [14].

Die übliche Stimulationsfrequenz von $130 \mathrm{~Hz}$ führt bei manchen Patienten mit Freezing nicht zu einer befriedigenden Besserung. Hier hat sich gezeigt, dass Frequenzen um $60-80 \mathrm{~Hz}$ eine bessere Linderung der Freezing Symptomatik bewirken können [15].

Ein weiterer Parameter neuerer THS-Systeme ist die Direktionalität der Stimulation aufgrund segmentierter Elektrodenkontakte. Dabei lässt sich das elektrische Feld horizontal der Elektrodenachse in drei Richtungen lenken, was die Programmieroptionen deutlich erweitert. Die direktionale Stimulation ermöglicht eine präzisere Ansteuerung der Zielstruktur, was klinisch das therapeutische Fenster vergrößert [16]. Die direktionale Stimulation führt bei gleicher Intensität zu einer stärkeren neuronalen Aktivierung als die omnidirektionale Stimulation [17]. Deshalb ist die Intensität bei Umstellung von einer omnidirektionalen auf eine direktionale Stimulation in der Regel zu reduzieren.

Generelles Ziel der Programmierung ist es, das elektrische Feld so an die Zielstruktur anzupassen und auf diese zu begrenzen, dass möglichst gute therapeutische Wirkungen erzielt und Nebenwirkungen durch eine unerwünschte Stimulation von Nachbarstrukturen vermieden werden. Insbesondere bei nicht wiederaufladbaren Systemen sollte die Programmierung auch den Stromverbrauch berücksichtigen, um eine möglichst lange Lebensdauer der Batterie zu gewährleisten.

\section{Merke}

Der beste Elektrodenkontakt für die chronische therapeutische Stimulation wird im monopolaren Review anhand der Größe des therapeutischen Fensters bestimmt. Das therapeutische Fenster ergibt sich aus der Differenz der Amplitudenschwellen für das Auftreten einer klinisch relevanten

Symptombesserung und das Auftreten relevanter Nebenwirkungen.

\section{Klinische Ergebnisse}

Eine Metaanalyse aus 37 unkontrollierten Studien [18] sowie 5 große randomisierte kontrollierte Studien [11, 1821] haben gezeigt, dass die STN-THS zu einer Reduktion der motorischen Symptome im Med-OFF (UPDRS III), einer Verbesserung der Aktivitäten des täglichen Lebens (UPDRS II), einer Verringerung von Dyskinesien im Med-ON (UPDRS IV), einer Verkürzung von OFF Phasen (Patiententagebuch), einer Verlängerung von ON Phasen ohne behindernde Dyskinesen (Patiententagebuch) und einer Zunahme der Lebensqualität führt (PDQ-39).

Unten sind für die unkontrollierten Studien der Metanalyse die prozentualen Veränderungen (Mittelwerte) unter Stimulation im Vergleich zu Baseline (linker Wert) und für die kontrollierten Studien die prozentualen Veränderungen (Mittelwerte) zwischen stimulierter Patientengruppe und nicht-stimulierter Patientenkontrollgruppe aufgeführt (rechter Wert).

\section{Merke}

Durch die STN-THS ist eine Besserung folgender

Outcome-Parameter zu erwarten [22]:

- Reduktion motorischer Symptome im Med-OFF: 52 / 35,4\%

- Verbesserung der Aktivitäten des täglichen Lebens: 49,9 / 33,0\%

- Verringerung von Dyskinesien im Med-ON ((UPDRS IV): 69,1 / 49,1\%

- Reduktion der OFF-Zeit: 68,2 / 50,8\%

- Verbesserung der Lebensqualität: 34,5 / 20,8\%

- Reduktion der täglichen L-Dopa Äquivalenzdosis: 55,9 / $37,0 \%$

\section{Stimulationsassoziierte Nebenwirkungen}

Neben den oben erwähnten operationsbedingten Risiken können bei der STN-THS stimulationsassoziierte Nebenwirkungen auftreten, die sich in der Regel durch die unerwünschte Mitstimulation von Nachbarstrukturen erklären lassen. So können innerhalb von Sekunden bis Stunden nach Einschalten der THS eine Dysarthrophonie, Gangstörungen, Augenbewegungsstörungen, unwillkürliche Muskelkontraktionen, Dyskinesien oder eine Lidöffnungsapra- 
xie auftreten. Eine häufige transiente Nebenwirkung nur beim Einschalten des Stimulators stellen Parästhesien dar, die bei einer zu weit nach posterior ausgerichteten Stimulation gelegentlich auch persistieren können. Stimulationsinduzierte Nebenwirkungen sind prinzipiell bei Reduktion der Amplitude reversibel und lassen sich somit durch eine Anpassung der Stimulationsparameter beherrschen.

Als kognitive Nebenwirkungen können leichte Störungen im Bereich der Wortflüssigkeit auftreten, die jedoch in der Regel keine Alltagsrelevanz und keinen Einfluss auf die Lebensqualität der operierten Patienten haben [23]. In den Wochen nach der Operation kann sich bei normaler Stimmung und guter Beweglichkeit eine Apathie einstellen, die auf eine zu starke Reduktion der dopaminergen Medikation zurückgeführt und entsprechend durch erneute Einführung oder Erhöhung eines Dopamin-Agonisten behandelt wird.

\section{KERNAUSSAGEN}

- STN-THS ist eine etablierte Therapie des fortgeschrittenen M. Parkinson.

- Die Indikationsprüfung sollte bei Vorhandensein behindernder motorischer Fluktuationen/ Dyskinesien und/oder therapierefraktärem Tremor erfolgen.

- Eine sorgfältige Patientenselektion ist eine wesentliche Voraussetzung für den Therapieerfolg.

- Das monopolare Review sollte nach Abklingen des Setzeffektes durchgeführt werden und dient der Auswahl des besten Kontaktes für die chronische Stimulation.

- Unter der STN-THS bessern sich in der Regel L-Dopa-responsive Symptome, Tremor, hypodopaminerge motorische und nicht-motorische Wirkfluktuationen sowie Dyskinesien und hyperdopaminerge neuropsychiatrische Symptome.

- Durch direktionale Stimulation mit segmentierten Elektroden kann das therapeutische Fenster der THS vergrößert werden.
Interessenkonflikt

\section{Erklärung zu finanziellen Interessen}

Forschungsförderung erhalten: nein; Honorar/geldwerten Vorteil für Referententätigkeit erhalten: nein; Bezahlter Berater/ interner Schulungsreferent/Gehaltsempfänger: nein; Patent/ Geschäftsanteile/Aktien (Autor/Partner, Ehepartner, Kinder) an im Bereich der Medizin aktiven Firma: nein. Patent/Geschäftsanteile/Aktien (Autor/Partner, Ehepartner, Kinder) an zu Sponsoren dieser Fortbildung bzw. durch die Fortbildung in ihren Geschäftsinteressen berührten Firma: nein.

Erklärung zu nichtfinanziellen Interessen

Deutsche Parkinson Gesellschaft

\section{Autorinnen/Autoren}

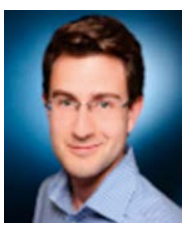

\section{Petyo Nikolov}

2011-2018 Studium der Humanmedizin an der Heinrich Heine Universität, Düsseldorf. Seit 2019 wissenschaftlicher Mitarbeiter am Institut für Klinische Neurowissenschaften und Medizinische Psychologie des Universitätsklinikums Düsseldorf.

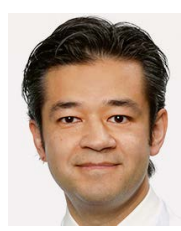

\section{Stefan Jun Groiss}

Oberarzt in der Klinik für Neurologie und wissenschaftlicher Mitarbeiter im Institut für Klinische Neurowissenschaften und Medizinische Psychologie, Heinrich Heine Universität, Düsseldorf.

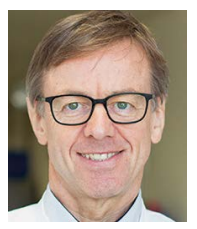

\section{Alfons Schnitzler}

Ärztlicher Leiter des Zentrums für Bewegungsstörungen und Neuromodulation der Klinik für Neurologie, Heinrich Heine Universität, Düsseldorf.

\section{Korrespondenzadresse}

Petyo Nikolov

Universitätsklinikum Düsseldorf

Moorenstraße 5

40225 Düsseldorf

Petyo.Nikolov@med.uni-duesseldorf.de

\section{Wissenschaftlich verantwortlich gemäß Zertifizierungsbestimmungen}

Wissenschaftlich verantwortlich gemäß Zertifizierungsbestimmungen für diesen Beitrag ist Prof. Dr. med. Alfons Schnitzler, Düsseldorf. 
Literatur

[1] Hartmann C], Fliegen S, Groiss S] et al. An update on best practice of deep brain stimulation in Parkinson's disease. Ther Adv Neurol Disord 2019; 12: 1756286419838096

[2] Fahn S, Oakes D, Shoulson I et al. Levodopa and the progression of Parkinson's disease. N Engl J Med 2004; 351: 2498-2508

[3] Ray Chaudhuri K, Poewe W, Brooks D. Motor and Nonmotor Complications of Levodopa: Phenomenology, Risk Factors, and Imaging Features. Mov Disord Off J Mov Disord Soc 2018; 33: 909-919

[4] Park K, Oeda T, Kohsaka M et al. Low body mass index and life prognosis in Parkinson's disease. Parkinsonism Relat Disord 2018; 55: 81-85

[5] Benabid AL, Pollak P, Gross C et al. Acute and long-term effects of subthalamic nucleus stimulation in Parkinson's disease. Stereotact Funct Neurosurg 1994; 62: 76-84

[6] McIntyre CC, Grill WM, Sherman DL et al. Cellular effects of deep brain stimulation: model-based analysis of activation and inhibition. J Neurophysiol 2004; 91: 1457-1469

[7] Gradinaru V, Mogri M, Thompson KR et al. Optical deconstruction of parkinsonian neural circuitry. Science 2009; 324: 354-359

[8] Oswal A, Litvak V, Sauleau P et al. Beta reactivity, prospective facilitation of executive processing, and its dependence on dopaminergic therapy in Parkinson's disease. J Neurosci Off J Soc Neurosci 2012; 32: 9909-9916

[9] Lhommée E, Wojtecki L, Czernecki V et al. Behavioural outcomes of subthalamic stimulation and medical therapy versus medical therapy alone for Parkinson's disease with early motor complications (EARLYSTIM trial): secondary analysis of an open-label randomised trial. Lancet Neurol. 2018; 17: 223-231. Im Internet: http://www.sciencedirect. com/science/article/pii/S1474442218300358

[10] Derost P-P, Ouchchane L, Morand D, Ulla M et al. Is DBS-STN appropriate to treat severe Parkinson disease in an elderly population? Neurology 2007; 68: 1345-1355

[11] Schuepbach WMM, Rau J, Knudsen K et al. Neurostimulation for Parkinson's disease with early motor complications. N Engl J Med 2013; 368: 610-622

[12] Fenoy AJ, Simpson RK. Risks of common complications in deep brain stimulation surgery: management and avoidance. J Neurosurg 2014; 120: 132-139

[13] Volkmann J, Herzog J, Kopper F et al. Introduction to the programming of deep brain stimulators. Mov Disord 2002; 17: S181-S187. Im Internet: https://onlinelibrary.wiley.com/ doi/abs/10.1002/mds.10162
[14] Dayal V, Grover T, Tripoliti E et al. Short Versus Conventional Pulse-Width Deep Brain Stimulation in Parkinson's Disease: A Randomized Crossover Comparison. Mov Disord Off J Mov Disord Soc 2020; 35: 101-108

[15] Moreau C, Defebvre L, Destée A et al. STN-DBS frequency effects on freezing of gait in advanced Parkinson disease. Neurology 2008; 71: 80-84

[16] Dembek TA, Reker P, Visser-Vandewalle V et al. Directional DBS increases side-effect thresholds-A prospective, double-blind trial. Mov Disord Off J Mov Disord Soc 2017; 32: 1380-1388

[17] Rebelo P, Green AL, Aziz TZ et al. Thalamic Directional Deep Brain Stimulation for tremor: Spend less, get more. Brain Stimulat. 2018; 11: 600-606. Im Internet: http://www. sciencedirect.com/science/article/pii/S1935861X17310318

[18] Deuschl G, Schade-Brittinger C, Krack P et al. A randomized trial of deep-brain stimulation for Parkinson's disease. N Engl J Med 2006; 355: 896-908

[19] Williams A, Gill S, Varma T et al. Deep brain stimulation plus best medical therapy versus best medical therapy alone for advanced Parkinson's disease (PD SURG trial): a randomised, open-label trial. Lancet Neurol 2010; 9: 581-591

[20] Okun MS, Gallo BV, Mandybur G et al. Subthalamic deep brain stimulation with a constant-current device in Parkinson's disease: an open-label randomised controlled trial. Lancet Neurol 2012; 11: 140-149

[21] Weaver FM, Follett K, Stern M et al. Bilateral deep brain stimulation vs best medical therapy for patients with advanced Parkinson disease: a randomized controlled trial. JAMA 2009; 301: 63-73

[22] Krack P, Volkmann J, Tinkhauser G et al. Deep Brain Stimulation in Movement Disorders: From Experimental Surgery to Evidence-Based Therapy. Mov Disord Off J Mov Disord Soc 2019; 34: 1795-1810

[23] Witt K, Daniels C, Reiff ] et al. Neuropsychological and psychiatric changes after deep brain stimulation for Parkinson's disease: a randomised, multicentre study. Lancet Neurol 2008; 7: 605-614

\section{Bibliografie}

DOI https://doi.org/10.1055/a-1046-0850

Klin Neurophysiol 2020; 51: 57-66

(c) Georg Thieme Verlag KG Stuttgart · New York ISSN 1434-0275 


\section{CME-Fragen bei CME.thienede}

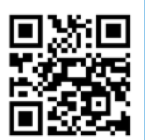

Diese Fortbildungseinheit ist bis zu 12 Monate online für die Teilnahme verfügbar.

Den genauen Einsendeschluss finden Sie beim Modul auf https://eref.thieme.de/CXE4786

Sollten Sie Fragen zur Online-Teilnahme haben, finden Sie unter https://cme.thieme.de/hilfe

eine ausführliche Anleitung. Wir wünschen viel Erfolg beim Beantworten der Fragen!

Unter https://eref.thieme.de/CXE4786 oder über den QR-Code kommen Sie direkt zum Artikel.

\section{Frage 1}

Was stellt eine gute Indikation zur STN-THS dar?

A Eine ausgeprägte Gangataxie

B Eine seit 2 Jahren bestehende Depression

C Motorische Fluktuationen unter L-Dopa Therapie

D Alter des Patienten > 75 Jahre

E Gute Symptomkontrolle unter medikamentöser Therapie

\section{Frage 2}

Welches Parkinsonsymptom kann erwartungsgemäß durch die THS gebessert werden?

A Nachlassen des Gedächtnisses

B L-Dopa resistenter Tremor

C Orthostatische Dysregulation

D Inkontinenz

E Verbale Flüssigkeit

\section{Frage 3}

Was stellt eine Kontraindikation zur THS bei M. Parkinson dar?

A Vorliegen einer Demenz

B Ausgeprägter Tremor

C Deutliche Dyskinesien unter L-Dopa Einnahme

D Vorliegen einer Polyneuropathie

E Freezing of Gait

\section{Frage 4}

Welche Aussage ist richtig?

A Motorische Fluktuationen treten im fortgeschrittenen Parkinsonstadium immer ab 8 Jahren Krankheitsdauer auf

B Freezing of gait stellt eine Kontraindikation zur THS dar

C STN-THS kann das Auftreten von Impulskontrollstörungen und Halluzinationen durch Reduktion der dopaminergen Medikation vermindern

D Nach STN-THS wird oft eine Erhöhung der L-Dopa Einnahme erforderlich (synergistischer Effekt)

E Die Lebensqualität wird von THS insgesamt nicht beeinflusst

\section{Frage 5}

Welche Aussage ist richtig?

A Eine Apathie könnte in den ersten Wochen nach der Operation, vor allem bei starker L-Dopa Reduktion auftreten

B Stimulationsinduzierte Nebenwirkungen sind irreversibel

C Parästhesien sind ein sicheres Zeichen, dass die Stimulation zu weit nach anterior ausgerichtet ist

D Die Mikroelektrodenableitung (MER) erfolgt 2-3 Tage nach der Operation

E Der GPi spielt aktuell keine Rolle mehr als Zielstruktur für die THS bei Parkinson

\section{Frage 6}

Welche Aussage ist falsch?

A Für die anatomische Zielpunktbestimmung und Trajektplanung wird eine Fusion von MRT und stereotaktischer CT Bildgebung benutzt.

B Ein unter L-Dopa nicht gebesserter Tremor reagiert auch nicht auf THS

C Die Mikroelektrodenableitung (MER) und die intraoperative Teststimulation dienen als Unterstützung zur Zielpunktfindung beim Einsetzen der Elektrode

D Das monopolare Review sollte am besten nach Abklingen des Mikroläsionseffektes durchgeführt werden

E Der STN wird in Europa dem GPi als Zielstruktur für die THS bei Parkinson bevorzugt

\section{Frage 7}

Was ist bei einer STN-THS nicht zu erwarten?

A Eine Besserung der motorischen Kardinalsymptome (Bradykinese, Rigor, Tremor)

B Eine mögliche Verschlechterung der verbalen Flüssigkeit

C Eine Besserung der Lebensqualität

D Eine Reduktion der täglichen L-Dopa Dosis

E Eine Besserung der kognitiven Leistung 


\section{CME-Fragen bei CME.thieme.de}

Fortsetzung ...

\section{Frage 8}

Welche Aussage über die direktionale Stimulation ist richtig?

A Die direktionale Stimulation ist der omnidirektionalen Stimulation bezüglich des klinischen Outcomes unterlegen

B Die direktionale Stimulation ist mit einer größeren therapeutischen Fenster assoziiert

C Die direktionale Stimulation erfordert deutlich höhere Stimulationsamplitude als die omnidirektionale Stimulation

D Bei stimulationsassoziierter Dyskinesie ist eine Erhöhung der Stimulationsintensität sinnvoll

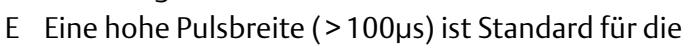
direktionale STN-THS

\section{Frage 9}

Welche Aussage bezüglich des monopolaren Reviews ist richtig?

A Das monopolare Review dient dazu, die richtige Pulsbreite zu bestimmen

B Das Ziel des monopolaren Reviews ist es, die besten Kontakte für die Dauerstimulation zu finden

C Beim monopolaren Review wird die Stimulationsfrequenz variiert

D Die besten Kontakte für die therapeutische Stimulation sind diejenigen mit dem kleinsten therapeutischen Fenster im monopolaren Review.

E Ein monopolares Review wird mit den modernen THS Systemen nicht mehr benötigt

\section{Frage 10}

Welche Aussage über stimulationsinduzierte Nebenwirkungen ist falsch?

A Stimulationsinduzierte Nebenwirkungen können sofort oder verzögert auftreten

B Eine mögliche stimulationsinduzierte Nebenwirkung sind Doppelbilder

C Eine häufige stimulationsinduzierte Nebenwirkung ist Dysarthrie

D Bei stimulationsinduzierten Nebenwirkungen sollten die Elektroden explantiert werden

E Bei Auftreten stimulationsinduzierter Nebenwirkungen kann die Anwendung kürzerer Pulsbreiten sinnvoll sein. 